Palabra Clave (La Plata), octubre 2017, vol. 7, n 1, e035. ISSN 1853-9912

Universidad Nacional de La Plata.

Facultad de Humanidades y Ciencias de la Educación.

Departamento de Bibliotecología

\title{
Aproximaciones al estudio de contextos informacionales de instituciones cubanas del sector de educación superior
}

\section{Approaches to the study of informational contexts of Cuban institutions of higher education sector}

\author{
Gloria Ponjuán Dante *
}

* Universidad de La Habana. Facultad de Comunicación. Departamento de Ciencias de la Información, Cuba I gponjuan@infomed.sld.cu

\section{PALABRAS CLAVE}

Contextos

informacionales

Educación superior

Cuba

\section{KEYWORDS}

Informational

contexts

Higher education

Cuba

\begin{abstract}
RESUMEN
Objetivos: se pretende un acercamiento al comportamiento del contexto informacional de un conjunto de organizaciones cubanas que pertenecen al Sector de la Educación Superior. Metodología: se realizó un estudio exploratorio con una muestra de instituciones de información cubanas. Para este estudio se distribuyó una encuesta anónima a profesionales que trabajan en diferentes instancias informacionales de diferentes instituciones cubanas pertenecientes al sector de educación superior. Las respuestas fueron libres, o sea sin mediar un formato para marcar opciones, lo que permitió que se pronunciaran sin sesgo alguno acerca de los aspectos ya mencionados. Resultados: se aprecia una alta concentración de respuestas que ubican tanto a la gestión como a la Tecnología en el foco principal de atención para los participantes. Es de destacar que en estos dos temas existe un balance entre las miradas positivas y las negativas. Las soluciones propuestas guardan relación con los temas que se mencionan como negativos. No se apreciaron diferencias significativas con relación a otros sectores que se encuentran en fase de estudio en la actualidad. Se aprecia el predominio de los entornos reactivoadaptativos, al igual que en la población analizada, pero hay una reducción en la categoría inestableturbulento, por debajo de la población, que puede ser típica de un sector dedicado a la educación. Conclusiones: un análisis de los contextos puede contribuir a identificar aquellos aspectos que pueden tener una influencia positiva o negativa para un aspecto en particular, en este caso lo relativo a la información en las organizaciones, sugiriendo que la atención a su solución favorecería el funcionamiento del sistema y su efectividad. Algunas de las valoraciones positivas encuentran también lecturas negativas por parte de los encuestados, que buscan el perfeccionamiento de determinados aspectos dentro de temas más genéricos. Se emitieron recomendaciones a las instituciones participantes.
\end{abstract}

\section{ABSTRACT}

Objectives: an approach to the behavior of the informational context of a group of Cuban organizations belonging to the higher education sector is presented. Methodology: an exploratory study was developed using a simple of Cuban informational organizations belonging to the sector of higher education. An anonymous survey was carried out among professionals that work in different levels or roles in this sector. The answers were open, without preelaborated options, intending their pronunciation without a close redaction of the aspects considered. Results: a high concentration of answers is obtained corresponding to areas such as management or technology representing its importance to participants. The balance of positive and negative answers is interesting. The solutions proposed also are related to the negative answers obtained. No significant differences are preliminarily identified with other sectors being studied, but there is a reduction in the category unstable/turbulent that could be related to the characteristics of the educational sector. Conclusions: an analysis of the contexts can contribute to the identification of some items than can positively or negatively influence some particular issue, in this case information in organizations, suggesting that its attention can favor solutions in the system performance and its effectiveness. Some positive results also have negative views in some participants that look for an improvement of some items. Some recommendations were suggested to the participant's institutions

Recibido: 11 de mayo de 2017 | Aceptado: 15 de agosto de 2017 | Publicado: 9 de octubre de 2017 


\section{Introducción}

En las disciplinas que integran las ciencias de la información se hace referencia a la importancia que revisten los contextos organizacionales para su funcionamiento y desarrollo.

Sin embargo, son pocos los estudios específicos que analicen estos contextos y que valoren sus potencialidades o debilidades. En esta comunicación se pretende un acercamiento al comportamiento del contexto informacional de un conjunto de organizaciones cubanas del Sector de la Educación Superior, como parte de un estudio que pretenderá analizar posibles coincidencias o divergencias con otros sectores como la ciencia, la salud, la cultura y la prensa.

El término contexto 1 (del latín contextus; conocido también como contexto material y contexto abstracto o simbólico), es el conjunto de circunstancias (materiales o abstractas) que se producen alrededor de un hecho o evento dado, que están fiablemente comprobadas. También se le refiere como la agrupación de circunstancias específicas de lugar y tiempo, principalmente, en que se está produciendo el acto de la comunicación. Por eso la propia fuente enfatiza:

Las circunstancias que forman cada tipo de contexto son por lo general muy específicas y si bien pueden repetirse en otros momentos o lugares, es casi imposible que todas se agrupen del mismo modo, que tengan el mismo lugar o importancia que en otros casos, y también, que generen exactamente los mismos resultados.

Se desprende que el énfasis de este aspecto está en el conjunto de condiciones u elementos presente en este espacio o enclave, y el comportamiento de estas condiciones en su relación con los factores que tienen una determinada presencia; por lo tanto, a partir del contexto, se puede interpretar o entender un hecho.

En cuanto a la información, la propia Wikipedia plantea:

Cada usuario de la información tiene unas necesidades particulares, y unos condicionantes distintos. Esos condicionantes constituyen el contexto y se corresponderían con el entorno social, cognitivo, de capacitación informacional, etc. El uso que dé a la información dependerá del contexto de uso de la misma y este a su vez dependerá de circunstancias cambiantes, pues una de las características del contexto es su dinamismo.

Taylor (1982), en un artículo que mantiene su vigencia a pesar del tiempo transcurrido desde su generación, justificaba la importancia de los ambientes informacionales y aseveraba que la comprensión de ese ambiente le daría a los directivos una mejor apreciación del valor de la información en un contexto específico. Reconocía que solo el consumidor era el que podía asignar valor a un producto o servicio, al igual que un mensaje solo tenía valor en un contexto.

Este autor sugería que en un principio se podían examinar los ambientes informacionales y proponer una estructura que favoreciera su descripción, pues en ese contexto se generaban las tareas y los problemas. 
Identificaba tres ambientes informacionales:

a. Geográficos: definidos por un límite físico (por ejemplo: un vecindario, una ciudad, una región, un país, etc.).

b. Organizacionales: definida como una unidad social compleja, designada deliberadamente con propósitos específicos. En este contexto la organización puede ser parte de una organización mayor, como por ejemplo una división dentro de una corporación, un hospital, una firma consultora, un departamento de policía. En cada caso cumple con los criterios para los que fue gestado.

c. Social/intelectual/cultural: formado por un grupo de personas que tal vez se desconozcan entre sí, pero cuyos intereses profesionales o personales favorecen a diferentes productos o servicios de información. A nivel social, por ejemplo, los masones, los católicos, los numismáticos; a nivel intelectual, los químicos, los historiadores, los banqueros; a nivel cultural, los chicanos, los músicos de cámara, etc.

En 1986, Taylor expresaba que el análisis de los ambientes de uso de la información implicaba la descripción de organizaciones, personas y problemas y que era importante comprender la interface entre las personas y los sistemas, que denominó "espacio de negociación". También este espacio podía incluir consideraciones de carácter social o cultural.

Rowley (1998) distingue los contextos de los ambientes informacionales, al definirlos de la siguiente manera:

Ambiente informacional: es el que rodea a los contextos informacionales: está integrado por las fuerzas políticas, legales, regulatorias, sociales, económicas y tecnológicas.

Contexto informacional: es en el que se encuentran los sistemas de información. El contexto influye sobre el diseño del sistema y comprende o rodea al usuario. Las organizaciones constituyen una categoría importante de contexto, pero otros contextos también son válidos como la educación, la familia y la comunidad.

Según tal definición el ambiente ejerce influencia en el contexto, aunque a los efectos de lo planteado el objetivo principal de esta comunicación, teniendo en cuenta lo planteado por la autora, está en el contexto y no en el ambiente.

No obstante, cabe señalar que se ha observado que en la literatura de la especialidad se emplea en buena medida el concepto de ambiente sin considerar esta distinción que señala esta autora.

Otro concepto empleado es el llamado framework o marco, que tampoco hace referencia a la distinción entre contexto, ambiente o marco, aunque Rowley (1998) considera que tanto el contexto informacional como el ambiente informacional, junto a otros elementos 
como los sistemas de información y la recuperación de información, integran este marco para la gestión de la información.

Para esta autora, un contexto informacional es aquel espacio organizacional establecido por los límites instaurados por las políticas y regulaciones de la institución o país, donde se desarrollan procesos informacionales y de conocimiento en función del cumplimiento de la razón social de ese espacio. Por tanto, puede tratarse de una institución, de una comunidad, o de cualquier grupo que por razones sociales (edades, intereses, etc.) se agrupen con determinado objetivo.

Valentim (2010) ha estudiado los ambientes informacionales y organizacionales, estableciendo un vínculo con los flujos de información que se tipifican en función del nivel en que se desarrollan, por lo que identifica en una organización sus características a nivel estratégico, táctico y operacional.

\section{Metodología}

Se realizó un estudio exploratorio con una muestra de instituciones de información cubanas pertenecientes a instituciones de educación superior. Para este estudio se distribuyó una encuesta anónima donde se consideraban los siguientes elementos:

- Mención de los aspectos que influyen positivamente en el contexto informacional.

- Mención de los aspectos que influyen negativamente en el contexto informacional.

- Mención de tres soluciones prioritarias para mejorar el contexto informacional.

- Clasificación del contexto de acuerdo a las categorías propuestas por Mintzberg y Quinn (1993).

La muestra incluyó representantes de varios centros de educación superior de las provincias de Pinar del Rio, Habana, Villa Clara, Camagüey, Sancti Spíritus, Holguín y Santiago de Cuba, o sea, de 7 de las 14 provincias del país. Los encuestados trabajan en bibliotecas, son profesores de la especialidad de ciencias de la información en varios de estos centros, y/o especialistas de departamentos de información de centros de investigación de este sector.

Las respuestas fueron libres, es decir, sin mediar un formato para marcar opciones, lo que permitió que se pronunciaran sin sesgo alguno acerca de los aspectos ya mencionados. Para facilitar el estudio estas respuestas fueron agrupadas en categorías más amplias.

\section{Resultados y discusión}

Un resumen general de los aspectos positivos, negativos y propuestas de soluciones aparece en la tabla 1. 
Tabla 1. Resumen de las respuestas clasificadas en temas genéricos

\begin{tabular}{|l|c|c|c|}
\hline & Aspectos positivos & Aspectos negativos & Soluciones propuestas \\
\hline ALFIN & $1(2,04 \%)$ & $2(4 \%)$ & $7(14 \%)$ \\
\hline COMUNICACIÓN & $1(2,04 \%)$ & $4(8 \%)$ & $1(2 \%)$ \\
\hline CONTENIDOS & $2(4,08 \%)$ & $2(4 \%)$ & $1(2 \%)$ \\
\hline CULTURA & $2(4,08 \%)$ & $3(6 \%)$ & $2(4 \%)$ \\
\hline GESTIÓN & $32(65,30 \%)$ & $29(58 \%)$ & $29(58 \%)$ \\
\hline TECNOLOGIA & $11(22,44 \%)$ & $10(20 \%)$ & $10(20 \%)$ \\
\hline
\end{tabular}

La tabla anterior refleja la distribución total de los aspectos positivos y negativos identificados por los participantes, así como las soluciones propuestas para mejorar el contexto informacional de sus instituciones.

Se aprecia una alta concentración de respuestas que ubican tanto a la gestión como a la tecnología en el foco principal de atención para los participantes. Es de destacar que en estos dos temas existe un balance entre las miradas positivas y las negativas. Generalmente las soluciones propuestas guardan relación con los temas que se mencionan como negativos.

En el caso de la clasificación de los contextos de acuerdo a las categorías propuestas por Mintzberg, se presentan los siguientes resultados (tabla 2).

Tabla 2. Clasificación de los entornos informacionales del sector educación superior

\begin{tabular}{|c|c|}
\hline Categoría & Total \\
\hline Estable & $5(29,4 \%)$ \\
\hline Reactivo-adaptativo & $9(52,9 \%)$ \\
\hline Inestable-turbulento & $3(17,6 \%)$ \\
\hline
\end{tabular}

En este caso se aprecia el predominio de los entornos reactivo-adaptativos; la categoría inestable-turbulento puede ser típica de un sector dedicado a la educación.

\section{Análisis de los aspectos positivos}

Se recibieron un total de 49 opiniones positivas acerca de contextos informacionales.

Dentro de ellas, un $65 \%$ fueron de aspectos gerenciales y un $11 \%$ de aspectos relativos a la tecnología.

Dentro de los aspectos de gestión mencionados como positivos, se destaca, en primer lugar, el rol de las personas al mencionar: 


\begin{tabular}{|l|}
\hline Personal calificado \\
\hline Gestión de Información se lleva a cabo por profesionales de la información \\
\hline Consumo y retroalimentación informacional de estudiantes \\
\hline $\begin{array}{l}\text { Buena atención, preocupación y asesoramiento de los profesores de más } \\
\text { experiencia }\end{array}$ \\
\hline Experiencia de los especialistas en el trabajo bibliotecológico \\
\hline Personal que labora en el Departamento ha cursado estudios superiores \\
\hline Recursos humanos adecuados \\
\hline
\end{tabular}

En segundo lugar, se refleja lo relativo a las políticas:

La información es pública para los profesores del departamento

Transparencia en las informaciones sobre el cumplimiento de cada uno

Compartición y consenso en la información interna y corporativa

Establecimiento de las políticas y procedimientos para la administración y el uso de la información

Políticas adecuadas

En tercer lugar, aparece lo relativo a la cultura y competencias informacionales:

\begin{tabular}{|l|}
\hline Dominio del manejo de la información y cultura informacional \\
\hline Competencia del especialista en la gestión de información \\
\hline Competencias informacionales \\
\hline
\end{tabular}

En cuanto a la tecnología, en primer lugar se refieren a la infraestructura tecnológica disponible (Internet, intranet, etc.) y, en segundo lugar, a la disponibilidad de medios tecnológicos para el trabajo informacional.

\section{Análisis de los aspectos negativos}

En el caso de los contextos negativos se recibieron 50 valoraciones, también primando aquellas relativas a la gestión (58\%) y la tecnología (20\%). A juicio de los participantes, el más mencionado es el relativo a las políticas.

\begin{tabular}{|l|}
\hline No están documentadas las políticas \\
\hline Carencia de políticas \\
\hline Las políticas sobre Internet \\
\hline Las políticas organizacionales \\
\hline Políticas de Información \\
\hline Políticas desactualizadas en la biblioteca \\
\hline
\end{tabular}

En segundo lugar, dentro de los aspectos de gestión se mencionan 3 opiniones relativas a las deficiencias en la cultura informacional y a dificultades con los procesos.

\begin{tabular}{|l|}
\hline No existe una definición formal de los procesos \\
\hline No existe una información organizada \\
\hline
\end{tabular}

Dentro de las valoraciones negativas, después del tema de políticas mencionado como el principal dentro de gestión, le sigue, en segundo lugar, lo relativo a la disponibilidad de las tecnologías $y$, en tercer lugar, lo relativo a la comunicación organizacional. Es importante 
señalar que temas como la disponibilidad de tecnología, la cultura informacional y la comunicación organizacional, alcanzan el $50 \%$ de las opiniones generales analizadas y lo relativo a las políticas también alcanza una alta representatividad.

\section{Propuestas de soluciones}

Es interesante apreciar que entre las soluciones se contemplan temas como la Alfin, de tecnología y de contenidos, lo que puede interpretarse también como una aspiración al perfeccionamiento de los contenidos informacionales y su uso, al elevarse las competencias mediante acciones de Alfin. Esto se aprecia en la tabla 3.

Tabla 3. Soluciones propuestas para el sector de educación superior

\begin{tabular}{|c|c|}
\hline & Educación superior \\
\hline ALFIN & $7(14 \%)$ \\
\hline COMUNICACIÓN & $1(2 \%)$ \\
\hline CONTENIDOS & $1(2 \%)$ \\
\hline CULTURA & $2(4 \%)$ \\
\hline GESTIÓN & $29(58 \%)$ \\
\hline TECNOLOGÍA & $10(20 \%)$ \\
\hline
\end{tabular}

\section{Conclusiones}

Los resultados muestran cómo un análisis de los contextos puede contribuir a identificar aquellos aspectos que pueden tener una influencia positiva o negativa para un aspecto en particular; en este caso lo relativo a la información en las organizaciones de un sector tan relevante como la educación superior, sugiriendo que la atención a su solución favorecería el funcionamiento del sistema y su efectividad.

Algunas de las valoraciones positivas encuentran también lecturas negativas por parte de los encuestados, que buscan el perfeccionamiento de determinados aspectos dentro de temas más genéricos. Es decir, existen espacios que captan la atención de los miembros de la organización que los consideran vitales para el funcionamiento de las mismas. Hay que tener en cuenta que, al buscar como respuesta solo tres opciones, se intenta que las miradas se dirijan hacia aquellos temas que pueden estar causando mayores impactos en la organización.

Aun cuando el ensayo fue a pequeña escala, los resultados alcanzados son válidos y pueden ser trasladados a los decisores para sus valoraciones ulteriores.

Se recomienda ampliar el estudio en otros sectores para conocer el balance final de la importancia general de determinados indicadores y poder precisar si determinados planteamientos son particulares o son válidos para todo sector económico.

\section{Notas}

1 Contexto (2014, 11 de diciembre). En Wikipedia, La enciclopedia libre. Recuperado de: http://es.wikipedia.org/w/index.php?title=Contexto\&oldid=78714503 (20/12/2016) 


\section{Referencias bibliográficas}

Mintzberg, H., y Quinn, J. B. (1993). El proceso estratégico. Conceptos, contextos y casos. México: Prentice-Hall Interamericana.

Rowley, J. (1998). Towards a framework for information management. International journal of information management, 18(5), 359-369.

Taylor, R. S. (1982). Value-Added Processes in the Information Life Cycle. Journal of the American Society of Information Science, 33(5), 341-346

Taylor, R. S. (1986). Value-added processes in information systems. N.J.: Ablex.

Valentim, M. L. P. (2010). Ambientes e fluxos de informaçao. En Valentim, M.L.P. (org.) Ambientes e fluxos de informação (pp. 13-22). São Paulo: Cultura Acadêmica. 